\title{
Review of double mood stabilizer treatments for bipolar disorder in China
}

\author{
Weidong $\mathrm{Jin}^{1 *}$, Maria Uscinska ${ }^{2}$, Yongchun $\mathrm{Ma}^{2}$ \\ ${ }^{1}$ Department of Psychiatry, Tongde Hospital Affiliated to Zhejiang Chinese Medicine University (Zhejiang Province Tongde Hospit- \\ al, Zhejiang Province Mental Health Institute, Hangzhou, China \\ ${ }^{2}$ Institute of Psychiatry, Kings College London, London, UK \\ Email: ${ }^{*}$ wdjin@163.com
}

Received 6 May 2013; revised 5 June 2013; accepted 14 June 2013

Copyright (C) 2014 Weidong Jin et al. This is an open access article distributed under the Creative Commons Attribution License, which permits unrestricted use, distribution, and reproduction in any medium, provided the original work is properly cited. In accordance of the Creative Commons Attribution License all Copyrights (C) 2014 are reserved for SCIRP and the owner of the intellectual property Weidong Jin et al. All Copyright (C) 2014 are guarded by law and by SCIRP as a guardian.

\section{ABSTRACT}

Background: Although treatment guidelines for bipolar disorder in many countries commonly include lithium carbonate joint with sodium valproate, this combination is not effective for all patients. Moreover, some adverse reactions related to this treatment, neurotoxicity and interaction with other drugs justify and call for a new reviewing of the issue. Methods: Evidence base for the interactions of combined drugs, the metabolic features, action mechanism, efficacy and side effects of these treatment combinations were reviewed. Considerable attention was given to the relationship of the mutual action of these drugs with their clinical efficacy but also their side effects. Results: The efficacy of combination therapy of lithium with valproate for treatment and prevention of mania were superior to monotherapy of lithium or valproate. Conclusion: Double mood stabilizer therapy is the best relative treatment for patients with bipolar disorder, especially mania and related episodes.

\section{KEYWORDS}

\section{Bipolar Disorde; Double Mood Stabilizers; Lithium Carbonate; Sodium Valproate}

\section{INTRODUCTION}

The treatment of most manic, mixed and rapid cycling episodes involved a combination of mood stabilizers with antipsychotics. Although treatment guidelines for bipolar disorder in many countries commonly include lithium carbonate joint with sodium valproate, this com-

${ }^{*}$ Corresponding author. bination therapy has not been proven satisfactory for all patients. Such treatment combinations with their adverse reactions, neurotoxicity and interaction with other drugs justify and call for a new reviewing of the issue. The aims are to review the interactions of combined drugs, the metabolic features, action mechanism, curative as well as side effects of these treatment combinations. In this review, considerable attention is given to the relationship of the mutual action of these drugs with their clinical efficacy but also their side effects.

\section{THEORETICAL STUDY AND NEW CONCEPT OF DOUBLE MOOD STABILIZERS}

\subsection{Action Mechanisms of Mood Stabilizers}

Recent studies have revealed similarities as well as differences in the action mechanisms of mood stabilizers such as lithium carbonate and valproic acid sodium. These studies have shown that lithium might have a neuro-protective function that is attributed to its direct inhibition of the activity of GSK-3 $\beta$, and thus to the blocking the process of apoptosis facilitated by this enzyme. Furthermore, the same protective mechanism against damage to nerve cells caused by neurotoxicity of glutamic acid and cytotoxicity of $\mathrm{Ca}^{++}$overload, have been implemented in the effects of several other mood stabilizers such as valproic acid and lamotrigine. The inhibition of GSK-3 $\beta$ facilitated apoptosis by certain mood stabilizers, suggests a common treatment effect by coordinating the dysregulation of GSK-3 $\beta$. A further protective mechanism of nerves is associated with neurotrophic neuron function of valproic acid sodium and lithium carbonate [1]. 


\subsection{Investigation for Recommendations.}

Efforts have been made to identify the potential benefits of the combined treatment for bipolar disorder patients. An opinion survey was carried out among 70 psychiatric experts in China, with a respondence rate of $86.7 \%$. The results suggested that double mood stabilizers were suitable for conditions including treatment resistant manic episode, recurrent mania with longer duration, rapid cycling, mixed episode, mania persisting after standard therapy guidelines, serious mania and delirium mania. Furthermore, the respondents identified potential positive effects of the combination of these drugs on switching prevention, maintenance therapy, rapid effect, mixed and rapid cycling episodes. Importantly, there was a consensus among the psychiatrists that the combination therapy decreased the risk of lithium intoxication [2].

\subsection{The New Concept of Double Mood Stabilizers [3]}

Previously, certain types of bipolar disorder, especially severe manic episode, rapid cycling and mixed episode were managed by one mood stabilizer or a combination of a mood stabilizer with an antipsychotic. These treatments, however, proved to have low efficacy. Other treatments involving anticonvulsants, in particular two antiepileptic drugs were shown to be more effective. Clinical observation from series of cases suggested that the best combination of two mood stabilizers included lithium carbonate and an antiepileptic such as valproate. Consequently, current guidelines for the treatment of bipolar disorder in China recommend double mood stabilizers (the Chinese Bipolar Disorder Treatment Guideline).

\section{CLINICAL APPLICATIONS}

\subsection{Combination of Lithium Carbonate and Valproate Compared to Lithium Carbonate}

To explore the efficacy with side effects of the combination of lithium carbonate and valproate versus lithium carbonate in the treatment of mania, a clinical trial on 88 affected patients was conducted (Chinese Criteria of Classification and Diagnostic of Mental Disorder $3^{\text {rd }}$ (ccmd-3) [4]. Both groups were assessed for 8 weeks with BechRafaelsen mania rating scale (BMRS) and treatment emergent symptoms scale (TESS). The results of the BMRS revealed that patients who were given the combination therapy showed a significant improvement after the first week of treatment on items including verbal activity, flight of thoughts, hostility, mood, self-esteem and sleep disturbance. Moreover, patients that received lithium with valproate acid showed a significant improvement in symptoms compared to the group that received lithium carbonate only. An assessment of the combination treatment versus lithium after two weeks confirmed a significant improvement on items of verbal activity, noise level, social contact, and sexual activity. The same trend of change in symptoms was observed after four weeks, marked by decrease in motor activity, hostility, mood, self-esteem, social contact, sleep disturbance, sexual interest and verbal activity. With regard to the effect of lithium, no significant improvement in verbal activity and flight of thought was observed. Furthermore, there were no significant differences in side effects between the two treatment groups throughout the course of treatments. Thus, the study provided evidence that the combination of sodium valproate with lithium carbonate is more effective in improving the symptoms of mania compared to single lithium. This is consistent with outcomes of other six clinical trials, which commonly found the former treatment to be superior to the later [5].

Further research (Liu et al.) compared the efficacy of combination of intravenous valproate with oral lithium versus oral lithium mono-therapy on 40 patients with acute mania (CCMD-3). An assessment following the first week of treatment revealed a significant decrease in symptoms in the combination group compared to the single treatment. However, after the second week, a significant decrease was also observed in the lithium group. The differences in the changes of BMRS scores existed from first weekend to forth weekend. It has been indicted that the combination of lithium carbonate and sodium valproate can improve symptoms more rapidly than lithium [6].

An atypical antipsychotic is recommended when a mood stabilizer therapy does not lead to a complete resolution of mania symptoms. The efficacy of the combination of sodium valproate XR (Depakine), lithium carbonate and an atypical antipsychotic was assessed by Wang et al. The trial recruited 80 patients with manic episode (according to CCMD-3), 40 of which were treated with combination of atypical antipsychotics, Depakine and lithium carbonate, whereas the control group was treated with lithium and an atypical antipsychotic. Changes in symptoms were assessed during 6 weeks of treatment, using the Beck-Rafaelsen manic scale (BRMS). The results revealed a significant decrease in mania symptoms in both groups of patients, however, the improvement in the treatment group was more pronounced compared to the control group. Furthermore, the side effects did not differ between the two groups. In consideration of the above, the study recommended that the combination therapy for mania is more effective when used with an atypical drug [7].

\subsection{Combination of Lithium Carbonate and Valproate Compared to Valproate}

Ma et al. compared the efficacy and safety of combina- 
tion therapy of sodium valproate XR (Depakine), lithium carbonate and an atypical antipsychotic with the combination of sodium valproate XR (Depakine) and an antipsychotic [8]. Following six weeks of treatment the Beck-Rafaelsen manic scale (BRMS) revealed that both treatments led to significant improvement, however, the combination of two mood stabilizers with an antipsychotic showed more efficacy, without a significant difference in side effects between the treatment and control group.

\subsection{Combination of Lithium Carbonate and Valproate in Prevention}

The literature suggests that the combination of lithium and valproate might have an effect on better prevention of relapse of bipolar disorder.

Liu et al. compared the efficacy of lithium and valproate with the efficacy of lithium only in the prevention of bipolar disorder. In their study, 82 patients in remission of bipolar disorder were randomly assigned into treatment and control groups. Participants in the case group received the combination of the two drugs, whereas others in the control group received the monotherapy of lithium. After two years' follow up, a superior improvement rate in relapse prevention in the treatment group was $88.6 \%$ versus $68.4 \%$ in the control group. These results provided evidence supporting that lithium carbonate in combination with sodium valproate is more effective then lithium alone in prevention of bipolar disorder [9].

A similar study was carried out to compare the rates of relapse of bipolar disorder in patients treated with lithium, valproate and the combination of lithium with valproate over the period of five years ( $\mathrm{Xu}, 2007)$, which found that the relapse rates were significantly lower in the combination treatment group comparing to the control group [10]. In particular, the treatment of lithiumcombined with valproate was effective in preventing rapid cycling bipolar disorder [10].

\section{EVALUATION BY EBM [11]}

Finally, evidence based medicine research was conducted to draw a conclusion on the efficacy of double mood stabilizers (lithium carbonate and sodium valproate) relative to one mood stabilizer (lithium carbonate) in the treatment of bipolar disorder (Jin, 11). Six relevant studies were analyzed with regard to changes in symptoms, efficacy and remission rates. A significant improvement in symptoms between double and single mood stabilizer treatments was found (WMD: $-1.91,95 \% \mathrm{CI}$ : -2.83 $-1.00, \mathrm{Z}=4.10, \mathrm{P}<0.001)$. Furthermore, analysis revealed a significant improvement in rates of efficacy (81.8\% vs 65\%, OR = 2.29, 95\%CI: $1.30-4.75, \mathrm{Z}=2.76$, $\mathrm{P}<0.01$, and significant improvement in full remission rate $(56.6 \%$ vs $37.7 \%$, OR $=2.37,95 \% \mathrm{CI}: 1.33-4.24, \mathrm{Z}$ $=2.92, \mathrm{P}<0.01)$. Thus, the results strongly suggested that therapy which entailed two mood stabilizers is superior to a single mood stabilizer in the treatment and prevention of bipolar disorder (see Figure 1).

\section{THE INTERACTION BETWEEN TWO DURGS}

\subsection{Study on Animals [12]}

Jin et al. examined the effect of carbon valproate on the level of lithium serum in 20 rats. The rats were randomly allocated into two groups, and bred with $15 \mathrm{mg}$ lithium carbonate every day for 26 days. In addition, rats in the experimental group were bred with $10 \mathrm{mg}$ sodium valproate every day from the sixth day of lithium administration until the end of the study. To determine levels of lithium serum, tail blood samples were collected on the $5^{\text {th }}, 12^{\text {th }}, 19^{\text {th }}$ and $26^{\text {th }}$ day, using 909 electric determining instruments. With an exception of the $19^{\text {th }}$ day, there were no significant differences in serum lithium levels

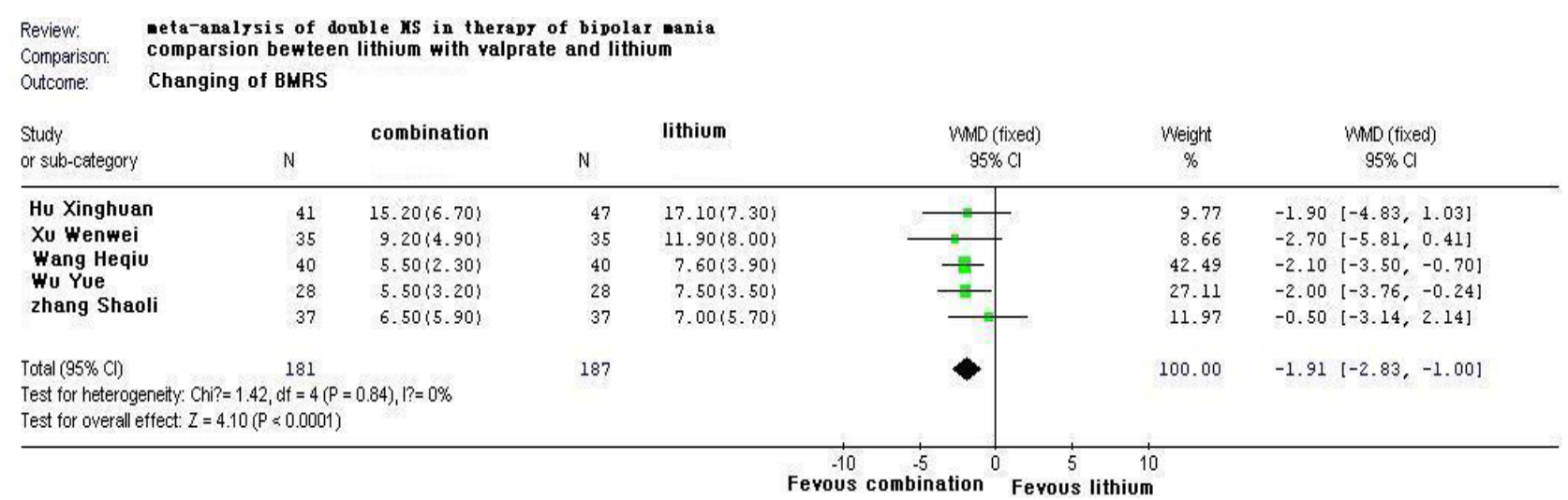

Figure 1. Manic symptoms change between lithium and combination of lithium and valpronate. Review: meta-analysis of double MS in therapy of bipolar mania; Comparison: comparison between lithium with valproate and lithium; Outcome: changing of BMRS. 
between the two groups. Thus, the research provided evidence that valproate does not influence the level of serum lithium and does not raise the level of lithium carbonate in serum.

\subsection{Clinical Evidence [13]}

A clinical trial was set out to investigate the mutual influence of serum concentration of lithium carbonate and sodium valproate (Jin, Ma, Ren, Wang, Xing and Tong, 2011). A total of 24 patients with bipolar mania, schizoaffective disorder and schizophrenia accompanied by excited agitation were randomly divided into 3 groups (n $=8$ per group). Apart from antipsychotics being administered to all patients, the first group received lithium carbonate; the second group was treated with sustained release sodium valproate and lithium carbonate; whereas the third group received sustained release sodium valproate at a constant dose for 1 week. The concentrations of drugs in serum were measured three times and revealed no significant differences in their levels between the three groups. Thus, the research provided further evidence supporting that mutual influence of lithium carbonate and sodium valproate was not significant.

\section{CONCLUSION}

In response to low efficacy of previous treatments for bipolar disorder, we reviewed the efficacy and safety of the combination of lithium carbonate and valproate, relative to other available treatments. Provided evidence supported that the combination under investigation is more effective and rapid in improving the symptoms of mania compared with any other single therapy. The review further revealed that lithium carbonate with sodium valproate was more effective than lithium alone in prevention of bipolar disorder, particularly in rapid cycling bipolar [10]. With regard to side effects, the combination of interest was equally safe to compared treatments.

\section{REFERENCES}

[1] Jin, W.D., Chen, J., Tang, X., Chen, Z., Zhang, J. and Tong, Z. (2007) Can two kinds of mood stabilizers share? Chinese psychiatric expert opinion surveys. Journal of Clinical Psychiatry, 17, 63-64. (in Chinese)

[2] Jin, W.D., Chen, Z. and Chen, J. (2007) Advances in the pharmacology and combination application of lithium carbonate and sodium valproate. Chinese Journal of Psychiatry, 40, 120-122. (in Chinese)

[3] Lu, W.M., Jin, W.D. and Wang, H., (2004) Double mood stabilizers for bipolar disorder: 3 case reports. Journal of Nervous Disorder and Mental Health, 4, 492. (in Chinese)

[4] Hu, X. and Gao, C.G. (2006) A Comparative study of double mood stabilizers in treating manic symptoms. Practice Journal of Medicine and Pharmacology, 23, 778-780. (in Chinese)

[5] Wu, Y., Lin, Y. and Li, Y. (2008) Control study of lithium carbonate combined with sodium valproate in the treatment of acute manic episodes. Journal of Clinical Psychology Medicine, 18, 51-52. (in Chinese)

[6] Liu, S., Tian, B. and Qi, W. (2011) A comparative study of intravenous valproate and lithium in the treatment of acute mania. Journal of Psychiatry, 24, 22-24. (in Chinese)

[7] Wang, H., Wang, N., Jin, W.D., Chen, J., Feng, B., Tong, Z. and Liu, L. (2008) Comparative study of combination of Sodium Valproate XR (Depakine) and Lithium Carbonate in the therapy for manic episodes. Journal of Nervous Disorder and Mental Health, 34, 98-100. (in Chinese)

[8] Ma, Y.C., Ren, Z.B., Jin, W.D., Wang, H., Xing, B., Chen, J. and Wang, N. (2010) Comparative study of atypical antipsychotics combination with double mood stabilizers in the therapy for manic episodes. Shanghai Archives of Psychiatry, 22, 165-167. (in Chinese)

[9] Liu, Y., Liu, W., Zhu, W. and Sun, L. (2007) A comparative study of lithium carbonate combined with sodium valproate in the prevention of bipolar disorder. Journal of Psychiatry, 20, 89-90.

[10] Xu, W., Wang, X., Chen, C. and Li, Y. (2007) Efficacy and safety of combination therapy of valproate with lithium for recurrent mania. Chinese Journal of Psychiatry, 40, 86-89. (in Chinese)

[11] Jin, W.D., Wang, H. and Wang, N. (2009) Meta-analysis of clinical efficacy of double mood stabilizers in treatment of bipolar disorder. Journal of Evidence-Based Medicine, 9, 168-171. (in Chinese)

[12] Jin, W.D., Wang, H., Zhang, J., Ma, Y., Lu, W., Chen, J., Tong, Z. and Xing, B. (2007) Study of valproate effects on serum lithium levels in rats. Chinese Journal of Clinical Pharmacy, 16, 116-118. (in Chinese)

[13] Jin, W.D., Ma, Y., Ren, Z., Wang, H., Xing, B. and Tong, Z. (2011) Mutual influence of drug concentration in serum between lithium carbonate and sodium valproate: A preliminary study. Chinese Journal of Psychiatry, in Press. (in Chinese) 